\title{
Genetics of Diabetic Retinopathy
}

\author{
Heeyoon Cho, MD, PhD ${ }^{1,2}$ and Lucia Sobrin, MD, MPH ${ }^{2}$ \\ Heeyoon Cho: hycho@hanyang.ac.kr; Lucia Sobrin: Lucia_Sobrin@meei.harvard.edu \\ ${ }^{1}$ Hanyang University College of Medicine, Department of Ophthalmology, 222 Wangsimni-ro, \\ Seongdong-gu, Seoul, 133-791, Korea (Republic of Korea), Tel. 82-31-560-2353, Fax \\ 82-31-564-9479 \\ ${ }^{2}$ Massachusetts Eye and Ear Infirmary, 243 Charles Street, $12^{\text {th }}$ floor, Boston, MA 02114, Tel. \\ 617-573-4279, Fax 617-573-3011
}

\begin{abstract}
Diabetic retinopathy (DR) is a polygenic disorder. Twin studies and familial aggregation studies have documented clear familial clustering. Heritability has been estimated to be as high as $27 \%$ for any DR and 52\% for proliferative diabetic retinopathy (PDR), an advanced form of the disease. Linkage analyses, candidate gene association studies and genome-wide association studies (GWAS) performed to date have not identified any widely reproducible risk loci for DR. Combined analysis of the data from multiple GWAS is emerging as an important next step to explain the unaccounted heritability. Key factors to future discovery of the genetic underpinnings of DR are precise DR ascertainment, a focus on the more heritable disease forms such as PDR, stringent selection of control participants with regards to duration of diabetes, and methods that allow combination of existing datasets from different ethnicities to achieve sufficient sample sizes to detect variants with modest effect sizes.
\end{abstract}

\section{Keywords}

Diabetic retinopathy; proliferative diabetic retinopathy; genetics; heritability; linkage studies; candidate gene association studies; genome wide association studies

\section{Introduction}

Diabetic retinopathy (DR) is the leading cause of blindness in Americans between 20-74 years of age.[1] The frequency and severity of DR among patients with diabetes mellitus is heterogeneous.[2-3] Known risk factors, most notably duration of diabetes and glycemic control, explain some, but not all, of the observed heterogeneity.[3-5] There are diabetic patients that develop DR despite a short durations of diabetes and/or excellent glycemic

Correspondence to: Lucia Sobrin, Lucia_Sobrin@meei .harvard.edu.

Conflict of Interest

Heeyoon Cho and Lucia Sobrin declare that they have no conflict of interest.

Compliance with Ethics Guidelines

Human and Animal Rights and Informed Consent

This article does not contain any studies with human or animal subjects performed by any of the authors. 
control and other diabetic patients who do not develop DR in the face of long standing diabetes and/or long-term hyperglycemia.[2] Genetic variation may explain some of the remaining heterogeneity in DR development.

A rapid expansion of research on the genetic determinants of DR has been seen in past two decades. Clinical and epidemiological studies have identified a genetic component to DR, but data from studies aimed at identifying genes or genome regions associated with DR have been quite inconsistent.[6-10*,**] The variability in results is probably due to several factors including differences in the ascertainment of retinopathy, different definitions of retinopathy, diverse control definitions with regards to duration of diabetes, and inadequate sample sizes. The purpose of this review is to summarize the findings of investigations aimed at uncovering the genetic underpinnings of DR and discuss current efforts in the field.

\section{Heritability and Linkage studies of diabetic retinopathy}

The role of genetic factors in shaping susceptibility to DR has been known for many years. Family-based studies have indicated that DR susceptibility is heritable. There is a high concordance of DR severity among twins with both type 1 diabetes (T1D) and type 2 diabetes (T2D).[11] Depending on the DR phenotype and ethnic population examined, siblings and relatives of diabetic patients with DR have approximately a 2- to 3-fold risk of DR compared with relatives of diabetic patients without DR.[12-18] The degree of familial aggregation is greater for more severe forms of retinopathy. Heritability has been estimated to be as high as $27 \%$ for DR and $52 \%$ for proliferative diabetic retinopathy (PDR), a more advanced form of the disease.[13-14]

In the 1980s and 1990s, the dominant study design for examining the genetic basis of pathological traits has been linkage analysis in families. This type of strategy is based on a search for regions in the genome with more than the expected number of shared alleles among affected individuals within a family. Linkage is quantified using a logarithm of odds (LOD) score with a LOD score of 3 or greater generally considered significant for evidence of linkage. Imperatore and colleagues conducted a sib-pair linkage analysis for DR in Pima Indians with T2D and found only modest evidence of linkage at chromosomes 3 and 9 with LOD scores of 1.36 and 1.46, respectively.[19] A subsequent genome-wide linkage analysis in this population found a stronger evidence for linkage on chromosome $1 \mathrm{p}$ with LOD scores of 2.58 and 3.1 for single-point and multi-point analyses, respectively.[14] Hallman et al. conducted a genome-wide linkage scan for genes contributing to DR using 794 diabetes participants from 393 Mexican-American families from Starr County, Texas, having at least two diabetic siblings.[20] Unconditional linkage analysis revealed only suggestive evidence of linkage with retinopathy on chromosomes 3 and 12.

In summary, linkage studies have yielded limited, inconsistent information about potential genetic loci for DR. This is not unexpected, as linkage studies are an optimal study design for Mendelian, or monogenic, diseases and have had limited success in complex, or polygenic, diseases such as DR. As opposed to monogenic diseases for which there is no significant environmental influence on disease and one rare variant is usually causative, polygenic diseases have environmental risk factors and are thought to be caused by many, 
more common variants which each contribute a small amount of risk to disease development. Linkage analyses are primarily successful when the causal variant has a large effect size, as in monogenic diseases. In contrast, clinic- or population-based association studies have proven a successful study design for polygenic diseases where effect sizes of the individual causal variants are small[21] and have been the primary approach utilized in DR.

\section{Candidate gene association studies}

Association studies for DR thus far have predominantly been candidate gene association studies. This study design typically selects participants with and without the disease of interest and compares the frequency of variants with some biologic rationale for a role in the disease between the two groups. Genes that have a putative role in DR and have been investigated by several groups include vascular endothelial growth factor (VEGF) gene, aldose reductase $(A L R)$ gene, receptor for advanced glycation end-products $(R A G E)$ gene, and genes in the renin-angiotensin system.[7] Despite a large number of candidate gene studies on DR, no consistent genetic associations with DR have been found using this method. The results from these studies have been well summarized in other reviews. [6-7, 9]

Here we will highlight two candidate gene studies because of the strengths of their study design. The first investigation examined erythropoietin $(E P O)$, an angiogenic factor observed in the eye, and found for an association between the $\mathrm{T}$ allele of rs1617640 in the $E P O$ promoter and PDR.[22*] The strengths of this study were the large sample size ( $n=2572$ across three cohorts), examination of the DR phenotype with greatest heritability (PDR), stringent definition of controls as participants without retinopathy despite diabetes for at least 15 years, and consistency in the effect found across separate cohorts. A second, albeit smaller, study found the opposite allele of this same single nucleotide polymorphism (SNP) in EPO to be associated with DR risk[23]; additional studies are still needed to confirm this finding. The second candidate gene association study examined rs7903146 in TCF7L2, a consistent risk locus for T2D, and found an association with PDR in Caucasians with T2D that replicated in an independent cohort.[24] This study also had a reasonable sample size ( $\mathrm{n}=1139$ in the discovery and replication cohorts combined), used the PDR phenotype, defined controls stringently with a minimum diabetes duration of 15 years, and replicated the finding in an independent cohort. TCF7L2 had already been studied in DR with both positive [24-25*] and negative results,[26] and additional study in other T2D populations is warranted.

Reasons for the lack of overall success with the candidate gene approach include insufficient sample sizes to detect the expected modest effects, lack of comprehensive coverage of variation in the genes of interest, and incorrect hypotheses about which specific genes are involved in the disease process. In an effort to overcome some of these limitations, the Candidate Gene Association Resource (CARe) performed a candidate gene study for DR with a sample size larger than those previously used ( $n=8040$ including discovery and replication cohorts) and comprehensive coverage of variation in 2000 genes associated with cardiovascular, metabolic and inflammatory pathways.[27] By not choosing specific genes but rather examining pathways and appropriately correcting for multiple hypothesis testing, 
the investigation was less reliant on the accuracy of a priori hypotheses regarding the causal genes. In the discovery cohort, variants in P selectin (SELP) and iduronidase (IDUA) were significantly associated with DR after multiple-hypothesis testing correction, but these associations could not be replicated in independent cohorts. Of note an association between $E P O$ and DR consistent with that found by the Tong et al. study [22] was detected but the $\mathrm{P}$ value was just below the threshold for significance after correction for multiple hypotheses.

\section{Genome-wide association studies}

GWAS are the other type of genetic association study that has been employed in the field of DR genetics. They have certain advantages compared with candidate gene studies. In GWAS, SNPs across the entire genome are tested for association to disease. This approach does not rely on a hypothesis about what gene is associated to disease, and therefore is not limited by existing knowledge of biology which may be incomplete. The more recent genome-wide genotyping platforms also provide high density sampling of all common human genetic variation. However, large sample sizes are still required to detect the modest effect sizes that we expect. A stringent threshold for significance, which has been widely accepted to be $5 \times 10^{-8}$, must be met to declare an association and replication in independent cohorts is a standard in the field before an association is accepted as bona fide. GWAS have been quite successful in other complex diseases such as age-related macular degeneration (AMD), T2D, and diabetic nephropathy, and have revealed disease pathways that were not suspected prior to the GWAS discoveries.[28-34]

GWAS for DR, however, have also not produced any consistent risk loci. The results of these GWAS are summarized in Table 1. The first study was performed in a MexicanAmerican T2D population on one of the earlier versions of the genome-wide genotyping platforms which covered common human variation less well than the more recent iterations of the technology. None of the SNPs examined met the threshold for genome-wide significance when they compared 103 cases with moderate to severe nonproliferative diabetic retinopathy (NPDR) and PDR with 183 controls with no DR or early NPDR.[35]. The second study took place in a Caucasian T1D population with 973 severe retinopathy cases defined by a questionnaire history of DR laser treatment and 1856 controls who never received laser treatment.[36**] This study did not identify any loci that were genome-wide significant, but generated candidate loci that were subsequently examined in a replications in an independent sample.[37] None of these loci achieved genome-wide significance in the subsequent analysis. A third GWAS for DR in a Chinese T2D cohort with 174 NPDR and PDR cases and 675 controls with no DR reported several variants that were associated with genome-wide significant $P$ values.[38] However, this study examined multiple genetic models and did not correct for these additional hypotheses and there was no independent replication attempted. With appropriate correction for the number of models examined, the genome-wide significance does not remain. The most recent GWAS of DR was in Chinese participants and compared 570 controls defined as patients with T2D for more than 8 years without DR vs. 437 patients with PDR with T2D. [39**] The cases and controls were similar with regards to duration of diabetes and hemoglobin $\mathrm{A}_{1 \mathrm{c}}$, the two strongest environmental influences on DR, but the study did not reveal any genome-wide significant loci. 
There are several reasons why DR GWAS have yet to yield significant findings. First, the sample sizes were modest by GWAS standards. As previously discussed, the genetic effects in DR are likely to be modest, with odds ratios of $<1.2$, and thus require large sample sizes to be identified. In other diseases where GWAS has been successful, at least several thousand cases and controls have generally been required for reproducible discovery of associated variants.[29, 32] Second, some of the DR GWAS used case definitions that include milder degrees of retinopathy that bias the results towards the null. The diagnosis of DR is clinically defined by the presence of retinal microvascular lesions in patients with T1D or T2D but, these retinal lesions are not specific and may also be present in patients without DM or normal individuals.[17, 18] A case with minimal retinopathic changes may not represent DR. Cases that are defined as having more advanced levels of retinopathy are likely to avoid this bias. Third, the studies have not consistently defined controls strictly with regards to duration of diabetes. Duration of diabetes has a strong impact on development of retinopathy. A participant who has no retinopathy after two years of T2D may develop PDR in the future. Inclusion of participants with short durations of diabetes can lead to misclassification of controls and also bias results to the null. We recognize that recruitment of controls is difficult and so it poses a challenge for genetic studies of DR. [19] Ideally, controls would be participants with very long durations of diabetes and no retinopathy. These individuals are a small subset of the diabetic population. In studies to date, controls have often been defined more loosely than this to achieve a critical control sample size.

There are also important sources of heterogeneity that might contribute to inconsistencies of the findings between the studies. First, DR ascertainment was performed differently with one study relying on questionnaire documentation of eye laser treatment, which has been validated as a measure of severe retinopathy $[14,15][16]$, and others using fundus examination and/or photography. The gold standard for evaluating DR has been Early Treatment Diabetic Retinopathy Study (ETDRS) 7-standard field 30-degree stereoscopic color fundus photographs graded using the modified Airlie House classification. Nondilated, wide-field fundus photography with scanning laser ophthalmoscope technology has recently become widely used and found to be comparable to the dilated ETDRS photography protocol in the ability to detect DR lesions.[40] These methods increase the chances that media opacity, such as cataract which is more common in diabetic patients, does not limit gradability of photographs. They also capture peripheral DR lesions that may not be seen easily on examination of the posterior pole alone or limited-field, central photography. DR ascertainment with multi-field imaging of both eyes across all studies would likely lead to more consistent findings. Second, the various GWAS for DR have examined different case definitions including PDR, NPDR and diabetic macular edema (DME). In some studies, participants with NPDR were classified as controls whereas in other studies they were considered cases. With such heterogeneity in case-control definition, it is not surprising the findings have varied. Finally, one GWAS was performed in participants with T1D, while the other three focused on patients with T2D. While DR appears clinically similar in both forms of diabetes, there are some observed differences in the overall prevalence and severity of retinopathy between the two forms.[41] We expect 
variants that impact DR development will be common to both type of diabetes but it is possible that there is some heterogeneity in the genetic susceptibility by diabetes type.

There are several ongoing GWAS of DR around the world. These GWAS are aiming to overcome the limitations of previous GWAS in several ways. First, sample sizes are larger in the ongoing studies. Apart from simply recruiting more participants, studies have been able to combine data sets from diverse populations to increase sample sizes as the technical challenges to aggregating data from different ethnicities have been overcome. There are concerted attempts in these larger genomic studies to harmonize phenotyping and focus on the extreme and more heritable phenotypes including PDR. Finally, collaborative replication of findings between GWAS will be important for distinguishing true findings from false positives. Large-scale consortia have been successful in other diseases, including AMD and T2D.[29, 32, 42-45] We are hopeful that when these same strategies are employed in GWAS for DR, we will begin to uncover the genetic underpinnings of this disease.

\section{Next-generation sequencing}

Next-generation sequencing is a recent technologic advance that is having a deep impact on clinical diagnosis and mutation discovery, particularly in Mendelian diseases. Sequencing, or determination of every nucleotide within a DNA molecule, can be performed for a candidate gene, the whole exome (all the protein-coding regions of DNA) or the whole genome. Whole exome sequencing has been shown to be a powerful and cost-effective method for detection of disease variants underlying monogenic diseases.[46] The role of next-generation sequencing for polygenic disorders is slowly being uncovered. Its success is contingent on the hypothesis that rarer variants also contribute to genetic susceptibility in complex diseases. There have been some initial promising findings with this approach in other polygenic diseases. For example, Seddon et al. applied targeted next-generation sequencing to coding regions of candidate genes within AMD-associated loci and pathways. [47] They found significant association of disease with rare, missense alleles in three genes in the complement pathway with significant effect sizes (odds ratios ranging from 2.2 to 3.6). Similar approaches with targeted sequencing of genetic loci that have been replicated consistently in GWAS or whole exome sequencing might eventually prove useful in DR gene discovery as well.

\section{Pharmacogenetics}

Intravitreal injection of VEGF inhibitors is an efficacious pharmacologic treatment option for both DME and PDR.[48-50] Intravitreal or periocular corticosteroids have also had some limited success in the treatment of DME.[51] Response to treatment or risk of side effects with these agents could be influenced by individual genetic polymorphisms in each patient. These variants could be in genes associated with the disease itself or in genes associated in some way with drug pharmacokinetics. The field of pharmacogenetics seeks to uncover such associations. Studies of pharmacogenetics in DR have been limited. One recent report found an association of the VEGF $\mathrm{C} 634 \mathrm{G}$ polymorphism with response to therapy.[52] Patients were treated with intravitreal bevacizumab, an anti-VEGF agent, and their response to this drug was assessed by the change in visual acuity and in macular 
thickness. Thirty-six eyes were designated poor responders and 68 eyes were termed good responders. Participants with the CG and GG genotypes had a decreased chance of positive treatment outcomes compared to participants with the CC genotype. Pharmacogenetic discovery is still in its infancy in DR and larger studies are needed to firmly establish associations with drug response. The goal of finding genetic markers that would help physicians tailor treatment for DME and PDR and/or predict which patients will respond best to certain treatments is an important goal of genetic discovery in DR.

\section{Conclusions}

Our understanding of the genetics of DR is limited at the present time. While there is strong evidence to suggest that DR is a heritable trait, linkage studies, candidate gene association studies and GWAS have not yet revealed any reproducible loci for DR. GWAS has been a successful study design for other complex diseases. For GWAS to be successful in DR, larger sample sizes examining cases with severe DR phenotypes and controls with no DR despite long duration of diabetes are needed. DR ascertainment ideally should be done with multi-field photography of both eyes. Rigorous replication in independent data sets with standardized definitions of DR across studies will be key to separating false positive associations from true associations. International collaborations for GWAS that take these methodologic issues into account have great promise for helping us to better understand the underlying genetic architecture of DR susceptibility.

\section{References}

1. National Institute of Diabetes and Digestive and Kidney Diseases. National diabetes statistics fact sheet: general information and national estimates on diabetes in the United States. Bethesda, MD: U.S. Department of Health and Human Services, National Institute of Health; 2000. Publication No. 02-3892

2. Nathan DM. Long-term complications of diabetes mellitus. N Engl J Med. 1993; 328:1676-85. [PubMed: 8487827]

3. Klein R, Klein BE, Moss SE, Cruickshanks KJ. The Wisconsin Epidemiologic Study of Diabetic Retinopathy: XVII. The 14-year incidence and progression of diabetic retinopathy and associated risk factors in type 1 diabetes. Ophthalmology. 1998; 105:1801-15. [PubMed: 9787347]

4. Klein R, Klein BE, Moss SE, Cruickshanks KJ. Relationship of hyperglycemia to the long-term incidence and progression of diabetic retinopathy. Arch Intern Med. 1994; 154:2169-78. [PubMed: 7944837]

5. Klein R, Klein BE, Moss SE, Linton KL. The Beaver Dam Eye Study. Retinopathy in adults with newly discovered and previously diagnosed diabetes mellitus. Ophthalmology. 1992; 99:58-62. [PubMed: 1741141]

6•. Kuo JZ, Wong TY, Rotter JI. Challenges in elucidating the genetics of diabetic retinopathy. JAMA Ophthalmol. 2014; 132:96-107. Comprehensive review article of the challenges facing the field of diabetic retinopathy genetics. [PubMed: 24201651]

7••. Abhary S, Hewitt AW, Burdon KP, Craig JE. A systematic meta-analysis of genetic association studies for diabetic retinopathy. Diabetes. 2009; 58:2137-47. Thorough meta-analysis of many of the candidate gene studies performed for diabetic retinopathy. [PubMed: 19587357]

8. Omar AF, Silva PS, Sun JK. Genetics of diabetic retinopathy. Semin Ophthalmol. 2013; 28:337-46. [PubMed: 24138043]

9. Liew G, Klein R, Wong TY. The role of genetics in susceptibility to diabetic retinopathy. Int Ophthalmol Clin. 2009; 49:35-52. [PubMed: 19349785] 
10. Schwartz SG, Brantley MA Jr, Flynn HW Jr. Genetics and diabetic retinopathy. Curr Diabetes Rev. 2013; 9:86-92. [PubMed: 22950970]

11. Leslie RD, Pyke DA. Diabetic retinopathy in identical twins. Diabetes. 1982; 31:19-21. [PubMed: 6759208]

12. The Diabetes Control and Complications Trial Research Group. Clustering of long-term complications in families with diabetes in the diabetes control and complications trial. Diabetes. 1997; 46:1829-39. [PubMed: 9356033]

13. Hietala K, Forsblom C, Summanen P, Groop PH. Heritability of proliferative diabetic retinopathy. Diabetes. 2008; 57:2176-80. [PubMed: 18443200]

14. Looker HC, Nelson RG, Chew E, et al. Genome-wide linkage analyses to identify Loci for diabetic retinopathy. Diabetes. 2007; 56:1160-6. [PubMed: 17395753]

15. Rema M, Saravanan G, Deepa R, Mohan V. Familial clustering of diabetic retinopathy in South Indian Type 2 diabetic patients. Diabet Med. 2002; 19:910-6. [PubMed: 12421427]

16. Zhang X, Gao Y, Zhou Z, et al. Familial clustering of diabetic retinopathy in Chongqing, China, type 2 diabetic patients. Eur J Ophthalmol. 2010; 20:911-8. [PubMed: 20306445]

17. Hallman DM, Huber JC Jr, Gonzalez VH, et al. Familial aggregation of severity of diabetic retinopathy in Mexican Americans from Starr County, Texas. Diabetes Care. 2005; 28:1163-8. [PubMed: 15855583]

18. Arar NH, Freedman BI, Adler SG, et al. Heritability of the severity of diabetic retinopathy: the FIND-Eye study. Invest Ophthalmol Vis Sci. 2008; 49:3839-45. [PubMed: 18765632]

19. Imperatore G, Hanson RL, Pettitt DJ, et al. Pima Diabetes Genes Group. Sib-pair linkage analysis for susceptibility genes for microvascular complications among Pima Indians with type 2 diabetes. Diabetes. 1998; 47:821-30. [PubMed: 9588456]

20. Hallman DM, Boerwinkle E, Gonzalez VH, et al. A genome-wide linkage scan for diabetic retinopathy susceptibility genes in Mexican Americans with type 2 diabetes from Starr County, Texas. Diabetes. 2007; 56:1167-73. [PubMed: 17251272]

21. McCarthy MI, Abecasis GR, Cardon LR, et al. Genome-wide association studies for complex traits: consensus, uncertainty and challenges. Nat Rev Genet. 2008; 9:356-69. [PubMed: 18398418]

22•. Tong Z, Yang Z, Patel S, et al. Promoter polymorphism of the erythropoietin gene in severe diabetic eye and kidney complications. Proc Natl Acad Sci U S A. 2008; 105:6998-7003. Welldesigned, well-powered candidate gene study for proliferative diabetic retinopathy and end-stage diabetic nephropathy with replication in independent cohorts. [PubMed: 18458324]

23. Abhary S, Burdon KP, Casson RJ, et al. Association between erythropoietin gene polymorphisms and diabetic retinopathy. Arch Ophthalmol. 2010; 128:102-6. [PubMed: 20065225]

24•. Luo J, Zhao L, Chen AY, et al. TCF7L2 Variation and Proliferative Diabetic Retinopathy. Diabetes. 2013; 62:2613-7. Well-designed candidate gene study for diabetic retinopathy with independent replication. [PubMed: 23434931]

25. Ciccacci C, Di Fusco D, Cacciotti L, et al. TCF7L2 gene polymorphisms and type 2 diabetes: association with diabetic retinopathy and cardiovascular autonomic neuropathy. Acta Diabetol. 2012

26. Buraczynska M, Swatowski A, Markowska-Gosik D, Kuczmaszewska A, Ksiazek A. Transcription factor 7-like 2 (TCF7L2) gene polymorphism and complication/comorbidity profile in type 2 diabetes patients. Diabetes Res Clin Pract. 2011; 93:390-5. [PubMed: 21641671]

27. Sobrin L, Green T, Sim X, et al. Candidate gene association study for diabetic retinopathy in persons with type 2 diabetes: the Candidate gene Association Resource (CARe). Invest Ophthalmol Vis Sci. 2011; 52:7593-602. [PubMed: 21873659]

28. Visscher PM, Brown MA, McCarthy MI, Yang J. Five years of GWAS discovery. Am J Hum Genet. 2012; 90:7-24. [PubMed: 22243964]

29. Fritsche LG, Chen W, Schu M, et al. Seven new loci associated with age-related macular degeneration. Nat Genet. 2013; 45:433-9. 9e1-2. [PubMed: 23455636]

30. Neale BM, Fagerness J, Reynolds R, et al. Genome-wide association study of advanced age-related macular degeneration identifies a role of the hepatic lipase gene (LIPC). Proc Natl Acad Sci U S A. 2010; 107:7395-400. [PubMed: 20385826] 
31. Yu Y, Bhangale TR, Fagerness J, et al. Common Variants near FRK/COL10A1 and VEGFA are Associated with Advanced Age-related Macular Degeneration. Hum Mol Genet. 2011

32. Morris AP, Voight BF, Teslovich TM, et al. Large-scale association analysis provides insights into the genetic architecture and pathophysiology of type 2 diabetes. Nat Genet. 2012; 44:981-90. [PubMed: 22885922]

33. Chen W, Stambolian D, Edwards AO, et al. Genetic variants near TIMP3 and high-density lipoprotein-associated loci influence susceptibility to age-related macular degeneration. Proc Natl Acad Sci U S A. 2010; 107:7401-6. [PubMed: 20385819]

34. Sandholm N, Salem RM, McKnight AJ, et al. New susceptibility loci associated with kidney disease in type 1 diabetes. PLoS Genet. 2012; 8:e1002921. [PubMed: 23028342]

35. Fu YP, Hallman DM, Gonzalez VH, et al. Identification of Diabetic Retinopathy Genes through a Genome-Wide Association Study among Mexican-Americans from Starr County, Texas. J Ophthalmol. 2010; 2010

36••. Grassi MA, Tikhomirov A, Ramalingam S, et al. Genome-wide meta-analysis for severe diabetic retinopathy. Hum Mol Genet. 2011; 20:2472-81. Largest genome-wide association carried out to date for diabetic retinopathy. [PubMed: 21441570]

37. Grassi MA, Tikhomirov A, Ramalingam S, et al. Replication analysis for severe diabetic retinopathy. Invest Ophthalmol Vis Sci. 2012; 53:2377-81. [PubMed: 22427569]

38. Huang YC, Lin JM, Lin HJ, et al. Genome-wide Association Study of Diabetic Retinopathy in a Taiwanese Population. Ophthalmology. 2011; 118:642-8. [PubMed: 21310492]

39••. Sheu WH, Kuo JZ, Lee IT, et al. Genome-wide association study in a Chinese population with diabetic retinopathy. Hum Mol Genet. 2013; 22:3165-73. Genome-wide association study that accounted well for non-genetic risk factors (glycemic control and duration of diabetes). [PubMed: 23562823]

40. Silva PS, Cavallerano JD, Sun JK, et al. Nonmydriatic ultrawide field retinal imaging compared with dilated standard 7-field 35-mm photography and retinal specialist examination for evaluation of diabetic retinopathy. Am J Ophthalmol. 2012; 154:549-59. e2. [PubMed: 22626617]

41. Aiello LP, Cahill MT, Wong JS. Systemic considerations in the management of diabetic retinopathy. Am J Ophthalmol. 2001; 132:760-76. [PubMed: 11704039]

42. Yadav S, Cotlarciuc I, Munroe PB, et al. Genome-wide analysis of blood pressure variability and ischemic stroke. Stroke. 2013; 44:2703-9. [PubMed: 23929743]

43. Dichgans M, Malik R, Konig IR, et al. Shared genetic susceptibility to ischemic stroke and coronary artery disease: a genome-wide analysis of common variants. Stroke. 2014; 45:24-36. [PubMed: 24262325]

44. Andreassen OA, McEvoy LK, Thompson WK, et al. Identifying Common Genetic Variants in Blood Pressure Due to Polygenic Pleiotropy With Associated Phenotypes. Hypertension. 2014

45. Tang H, Wei P, Duell EJ, et al. Genes-Environment Interactions in Obesity- and DiabetesAssociated Pancreatic Cancer: A GWAS Data Analysis. Cancer Epidemiol Biomarkers Prev. 2014; 23:98-106. [PubMed: 24136929]

46. Wang Z, Liu X, Yang BZ, Gelernter J. The Role and Challenges of Exome Sequencing in Studies of Human Diseases. Front Genet. 2013; 4:160. [PubMed: 24032039]

47. Seddon JM, Yu Y, Miller EC, et al. Rare variants in CFI, C3 and C9 are associated with high risk of advanced age-related macular degeneration. Nat Genet. 2013; 45:1366-70. [PubMed: 24036952]

48. Schmidt-Erfurth U, Lang GE, Holz FG, et al. Three-Year Outcomes of Individualized Ranibizumab Treatment in Patients with Diabetic Macular Edema: The RESTORE Extension Study. Ophthalmology. 2014

49. Brown DM, Nguyen QD, Marcus DM, et al. Long-term outcomes of ranibizumab therapy for diabetic macular edema: the 36-month results from two phase III trials: RISE and RIDE. Ophthalmology. 2013; 120:2013-22. [PubMed: 23706949]

50. Nicholson BP, Schachat AP. A review of clinical trials of anti-VEGF agents for diabetic retinopathy. Graefes Arch Clin Exp Ophthalmol. 2010; 248:915-30. [PubMed: 20174816] 
51. Qi HP, Bi S, Wei SQ, Cui H, Zhao JB. Intravitreal versus subtenon triamcinolone acetonide injection for diabetic macular edema: a systematic review and meta-analysis. Curr Eye Res. 2012; 37:1136-47. [PubMed: 22793880]

52. El-Shazly SF, El-Bradey MH, Tameesh MK. Vascular endothelial growth factor gene polymorphism prevalence in patients with diabetic macular edema and its correlation with antiVEGF treatment outcomes. Clin Experiment Ophthalmol. 2013 


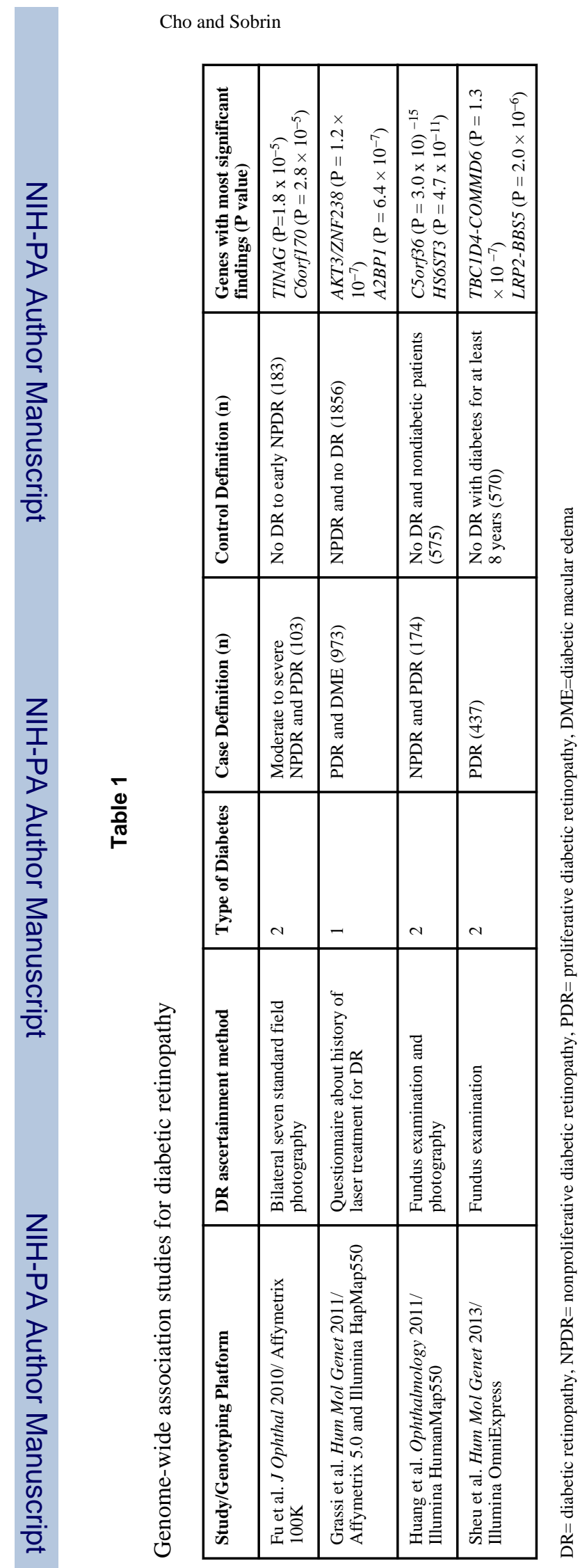

Page 11

(1)

Curr Diab Rep. Author manuscript; available in PMC 2015 August 01. 